\title{
Community health insurance in Uganda: status, obstacles and prospects
}

\author{
Robert Basaza \\ Supervisor: Patrick Van der Stuyft (1) \\ Co-supervisor: Bart Criel (2) \\ (I) Department of Public Health, Ghent University \\ (2) Department of Public Health, Institute of Tropical Medicine, Antwerp
}

Public defence: 19 May 2011

The estimated per capita annual health expenditure adopted in 2010 Uganda Country health financing review by a combined team of Ministry of Health, WHO Country office and Geneva was US\$27; the government and donors accounted for half of this and another half came from private out-of-pocket spending. The funding is inadequate compared to the US\$ 44 advocated by WHO. Amid political pressure and the exclusion of access to care of more than $50 \%$ of the population, user fees were abolished in 2001 from all publicly owned general wings of hospitals and health centres, although they continued to be levied in private wings. The private sector has always charged user fees and continues to do so. As a result, many patients still have difficulty paying bills from hospitals served by private, not-for-profit facilities.

Community Health Insurance (CHI) schemes aim to improve financial access to health care. It is voluntary arrangements, organised at the community level, that target individuals employed in the informal sector. The schemes run on a non-profit basis and apply the basic principle of risk sharing with community participation in design and management.

The ruling party's presidential election manifesto (2006-20II) includes using $\mathrm{CHI}$ as a way to provide protection against catastrophic health expenditures, but the number of schemes and persons covered by CHI remains small in Uganda. The 2010/II-2019/20 national health policy lists $\mathrm{CHI}$ as a financing mechanism for the health sector. Currently, the health sector is in the process of drafting a law to provide for $\mathrm{CHI}$ development. No studies in Uganda or the East African region have explored the possible reasons for low enrolment in CHI. Uganda's interest in promoting $\mathrm{CHI}$ and abolishing user fees for publicly owned health units requires examination. The perceptions of the communities in which the schemes exist have never been fully explored and little is known about the perceptions and knowledge of $\mathrm{CHI}$ held by scheme members, managers of district health services and policy-makers.

The aim of this thesis is to contribute to the understanding of $\mathrm{CHI}$, both in general and in Uganda specifically. The general objective is to investigate the obstacles and prospects for CHI schemes in Uganda. The specific objectives are: (I) to study demand and supply side factors to explain the low levels of enrolment in existing CHI schemes; (2) to investigate the perceptions of $\mathrm{CHI}$ held by those enrolled and those not enrolled in the 
schemes; (3) to determine knowledge, understanding and perceptions of the relevance of $\mathrm{CHI}$ among policy-makers and health service managers; and (4) to formulate evidencebased policy recommendations for the Ugandan health sector.

We set the stage for this research by using secondary data sources to embark on a review of the context of CHI schemes in sub-Saharan Africa. Results indicate that the coverage rates of the target population remain low. Other findings suggest that there are five dimensions that seem to be relevant within the framework of the development of CHI: political, economic, social, technical and managerial contexts. We acknowledged the need for more contextualised analyses of both successful and less successful schemes to enhance our collective understanding of why $\mathrm{CHI}$ works in certain circumstances and why it fails in others. Therefore, we set out to investigate in detail the emerging features of CHI schemes in East Africa.

Schemes from three East African countries (Uganda, Kenya and Tanzania) were examined using a grid to analytically tabulate their features. The key descriptive findings are as follows: (I) the schemes' coverage within their catchment areas was small; (2) the schemes had been in existence for only a short time; (3) they were established with donor support; and (4) they provided both inpatient and outpatient benefits. The findings of this study underscore the role of the government in the promotion of $\mathrm{CHI}$. This is especially true for advocacy, as the government must pay the premiums for the poorest citizens and develop an enabling policy and legal framework. We also concluded that there is a need for primary data to understand the mix of factors involved in $\mathrm{CHI}$ schemes.

The key reasons for low enrolment in Uganda were explored through an in-depth study of two selected CHI schemes: Ishaka in the Bushenyi District and the Save for Health Scheme in the Luwero District. This study involved a review of the schemes' records, scheme, district and Ministry of Health $(\mathrm{MOH})$ headquarters' key informant interviews and exit polls. We interviewed both insured and uninsured patients. On the demand side, the following determinants were identified: (I) lack of basic information on the scheme's design and operation, limited understanding of the principles underlying $\mathrm{CHI}$, (2) limited community involvement, (3) lack of trust in the management of the schemes and (4) problems with individuals' ability to pay their insurance premiums. On the supply side, we identified the following explanations: (I) limited knowledge on the part of CHI health care providers and managers, as well as (2) the absence of a coherent policy framework for the development of $\mathrm{CHI}$.

These results were further validated through two in-depth studies that separately examined demand and supply factors. The schemes we studied with regard to the demand side were again the Ishaka and the Save for Health Scheme. The population utilised for this qualitative demand side study included CHI scheme members and non-members living in each scheme's catchment area. Focus group discussions and in-depth interviews were carried out. We found that for many people in the catchment areas ( $\mathrm{I}$ ) a lack of good information and understanding of the basic principles of $\mathrm{CHI}$ and the routine functioning of the schemes as reasons for not enrolling, (2) difficulty in paying the premiums, (3) poor quality of health care, (4) rigid enrolment 
requirements and (5) problems of trust were important factors preventing people from joining.

The views of the policy-makers and health service managers were investigated through a qualitative supply side study using semi-structured interviews with District Health Officers (DHOs) and the staff of the $\mathrm{MOH}$ headquarters. The main finding was a lack of accurate information and understanding of $\mathrm{CHI}$ activities on the part of the staff of the $\mathrm{MOH}$ headquarters and DHOs. Out-of-pocket expenditure remains an important feature of health care financing in Uganda, despite the blanket abolition of user fees for government facilities. $\mathrm{CHI}$ is perceived as a relevant policy option and a potential source of funds for health care. It is also considered a means of raising the quality of health care in both public and private health units. Our study highlights that the current ambiguity and contradictions in the Ugandan MOH's health financing policy are in urgent need of attention.

We conclude that the reasons for not joining $\mathrm{CHI}$ schemes in Uganda are quite similar to those identified in studies of West African schemes. Findings specific to the Ugandan context are: (I) inappropriate entrance rules; (2) a benefit package that creates a barrier to enrolment for those most in need; and (3) lack of involvement of first-line health units, which reduces the attractiveness of the $\mathrm{CHI}$ schemes. However, the main challenge that must be tackled by $\mathrm{MOH}$ is the policy dilemma: how to reconcile the Ugandan government's interest in promoting $\mathrm{CHI}$ with the decision to abolish user fees for public health units. This situation is further compounded by the fact that the abolition of user fees for government facilities does not mean that out-of-pocket payments have disappeared altogether. District staff operates in an ambiguous policy environment and, as a result, they cannot promote $\mathrm{CHI}$ and be obliged to advocate free health care at the same time. A priority for the Ugandan government, therefore, is to establish a consistent policy framework that will unambiguously situate the relevancy and role of $\mathrm{CHI}$ in the national health system. $\mathrm{CHI}$ schemes must operate within a broader policy context that takes into consideration the national health financing strategy and legal framework.

The following policy options are proposed: (I) $\mathrm{CHI}$ implementation continues to be restricted to the private, not-for-profit sub-sector, as is the case today; (2) the Ugandan health sector implements $\mathrm{CHI}$ in the public sector, which implicitly means abandoning the policy of the abolition of user fees and reintroducing user charges in the public sector; and (3) CHI temporarily fills the gaps in local health care delivery and in the current social health protection coverage. If this final option is implemented, pooled prepayments (instead of the current individual, out-of-pocket payments) should be organised to pay for services and activities prioritised by $\mathrm{CHI}$ members.

We recommend further research to complement the findings of the current study. First, full stakeholder analysis of $\mathrm{CHI}$, with a focus on the political economy of health care, has to be carried out. In addition, quantitative studies should be designed to determine whether and to what extent $\mathrm{CHI}$ schemes in Uganda provide financial protection and improved access to health care. Finally, the issue of trust in the development of Ugandan CHI merits more detailed investigation, and trust-building structures and practises should be tested. 